\title{
Creation of matter in the universe and groups of type
}

\section{E7}

\author{
Sergio Ferrara ${ }^{a, b, c}$ and Renata Kallosh ${ }^{d}$ \\ ${ }^{a}$ Physics Department, Theory Unit, CERN, \\ 1211 Geneva 23, Switzerland \\ ${ }^{b}$ INFN, Laboratori Nazionali di Frascati, \\ Via Enrico Fermi, 40, 00044 Frascati, Italy \\ ${ }^{c}$ Department of Physics and Astronomy, University of California, Los Angeles, \\ CA 90095-1547,U.S.A. \\ ${ }^{d}$ Physics Department, Stanford University, \\ Stanford, CA 94305-4060, U.S.A. \\ E-mail: sergio.ferrara@cern.ch, kallosh@stanford.edu
}

ABSTRACT: We relate the mechanism of matter creation in the universe after inflation to a simple and universal mathematical property of extended $N>1$ supergravities and related compactifications of superstring theory. We show that in all such models, the inflaton field may decay into vector fields due to a nonminimal scalar-vector coupling. This coupling is compulsory for all scalars except $N=2$ hyperscalars. The proof is based on the fact that all extended supergravities described by symmetric coset spaces $\frac{G}{H}$ have duality groups $G$ of type E7, with exception of $\mathrm{U}(p, n)$ models. For $N=2$ we prove separately that special geometry requires a non-minimal scalar-vector coupling. Upon truncation to $N=1$ supergravity, extended models generically preserve the non-minimal scalar-vector coupling, with exception of $\mathrm{U}(p, n)$ models and hyperscalars. For some string theory/supergravity inflationary models, this coupling provides the only way to complete the process of creation of matter in the early universe.

Keywords: Cosmology of Theories beyond the SM, Extended Supersymmetry, Supergravity Models

ARXIV EPRINT: 1110.4048 


\section{Contents}

1 Introduction 1

2 Extended $d=4 N>1$ supergravity 3

2.1 Groups of type E7 and compulsory non-minimal vector coupling in $N>1 \quad 4$

$2.2 \quad N=2 \quad 6$

2.2.1 $N=2$ special geometry and compulsory non-minimal vector coupling 6

2.3 All kinetic terms in $N>1$

3 Consistent truncations of $N>1$ to $N=1 \quad 8$

4 Discussion $\quad 9$

$\begin{array}{ll}\text { A Special geometry and groups of type E7 } & 11\end{array}$

\section{Introduction}

According to inflationary theory, all elementary particles populating our universe have been created as a result of the process of preheating and reheating of the universe after inflation. During inflation, the energy density of the universe is dominated by the energy of the inflaton field $\phi$. At the end of inflation, the inflaton field may rapidly transfer a part of its energy to other particles and fields in the process of preheating, a non-perturbative process which may occur due to a combination of parametric resonance, tachyonic instability, and rescattering of produced particles and waves. However, this process does not take away all energy of the inflaton filed, so it should be followed by reheating, a perturbative particle production and the inflaton decay $[1,2]$. Reheating leads to a complete decay of the inflaton field only if the inflaton field can actually decay to other particles, i.e. if the theory allows the process $\phi \rightarrow$ anything rather than some interaction $\phi+\phi \rightarrow$ anything. There are some theories where the decay $\phi \rightarrow$ anything is forbidden, reheating is incomplete, and the universe eventually becomes dominated by the oscillating inflaton field, or by other scalar fields produced during preheating. Such theories are cosmologically unacceptable. Therefore it it important to understand whether there are some deep theoretical reasons to expect the existence of interactions which could lead to a complete decay of the inflaton field and of all other scalar fields which could be produced at the end of inflation.

A complete decay of the scalar fields may occur due to the scalar-dependent vector coupling, which is possible in $N=1$ supergravity [3]:

$$
-\frac{1}{4}\left(\operatorname{Re} f_{\alpha \beta}(\varphi)\right) F_{\mu \nu}^{\alpha} F^{\beta \mu \nu}+\frac{i}{4}\left(\operatorname{Im} f_{\alpha \beta}(\varphi)\right) F_{\mu \nu}^{\alpha} \tilde{F}^{\beta \mu \nu} .
$$


Here the function $f_{\alpha \beta}(\varphi)$ is holomorphic. However, whereas in $N=1$ supergravity the dependence of $f_{\alpha \beta}(\varphi)$ on scalars is possible, it is not required. The preference is often given to the minimal, scalar-independent vector couplings, where

$$
\operatorname{Re} f_{\alpha \beta}(\varphi)=\delta_{\alpha \beta}, \quad \operatorname{Im} f_{\alpha \beta}(\varphi)=0 .
$$

Indeed, the Ockham's razor principle generally recommends, when faced with competing hypotheses that are equal in other respects, selecting the one that makes the fewest new assumptions. At the level of phenomenological $N=1$ supergravity the non-minimal vector coupling requires an unmotivated extra assumption about the function $f_{\alpha \beta}(\varphi)$ and should therefore be avoided, unless such a motivation is provided. Moreover, in some textbooks $N=1$ supergravity is presented only in the form (1.2), see e.g. [4].

It has been recently explained in [5] that in some models of inflation in supergravity which provide an arbitrary inflaton potential $[6,7]$ there is no decay route for the inflaton field, unless one involves the non-minimal vector coupling. An analogous situation with reheating was noticed earlier in the string theory modular inflation models [8-11] as well as in related supergravity inflation models $[12,13]$. In [8-11] it was explained that in string theory standard model particles can live on wrapped D7 branes. In such case the inflaton which is the combination of the 4-cycle volume modulus $Y$ and its axionic partner $X$ naturally couple to vectors

$$
Y F_{\mu \nu} F^{\mu \nu}, \quad X F_{\mu \nu} \tilde{F}^{\mu \nu} .
$$

This provides a possibility of the creation of matter after inflation in these models via the inflaton decay into vectors. However, it was not clear whether this is just a specific example of the non-minimal vector coupling, or a generic feature of all phenomenological $N=1$ supergravity models derived from string theory via compactification and/or from extended supergravities. Since this issue appears in many inflationary models based on supergravity, we decided to investigate it.

We would like to note here that the non-minimal coupling of vectors is always complemented by the Pauli couplings of the form [3]

$$
\frac{\partial f_{\alpha \beta}(\varphi)}{\partial \varphi^{i}} \bar{\chi}^{i} \sigma_{\mu \nu} \lambda^{\alpha} F^{\beta \mu \nu}
$$

which allow the decay of the fermions $\bar{\chi}^{i}$ into gaugino $\lambda^{\alpha}$ and a vector $F^{\beta \mu \nu}$. This is in addition to the usual gaugino-gravitino-vector Pauli terms. Therefore if the superheavy scalar fields in the inflaton multiplet may completely decay after inflation, the same conclusion will be valid for their fermionic superpartners.

The purpose of this note is to prove that the non-minimal scalar-dependent vector coupling is compulsory (except for hyperscalars) in all $N>1$ supergravities, that have scalars, and in related superstring theory compactifications. This provides a motivation to use such couplings in phenomenological $N=1$ models inspired by the superstring theory/extended supergravity. We will also prove that generic $N>1$ supergravities consistently truncated to $N=1$ supergravity forbid the minimal choice (1.2). The only exception are $\mathrm{U}(p, n)$ models and the ones where scalars originate from $N=2$ hypers. 


\begin{tabular}{|c||c|c|}
\hline$N$ & $G$ & $\mathbf{R}$ \\
\hline \hline$N=3$ & $\mathrm{U}(3, n)$ & $(\mathbf{3}+\mathbf{n})$ \\
\hline$N=4$ & $\mathrm{SL}(2, \mathbb{R}) \otimes \mathrm{SO}(6, n)$ & $(\mathbf{2 , 6 + n})$ \\
\hline$N=5$ & $\mathrm{SU}(1,5)$ & $\mathbf{2 0}$ \\
\hline$N=6$ & $S O^{*}(12)$ & $\mathbf{3 2}$ \\
\hline$N=8$ & $E_{7(7)}$ & $\mathbf{5 6}$ \\
\hline
\end{tabular}

Table 1. $\quad N \geqslant 3$ supergravity sequence of groups $G$ of the corresponding $\frac{G}{H}$ symmetric spaces, and their symplectic representations $\mathbf{R}$

\section{Extended $d=4 N>1$ supergravity}

The bosonic part of $d=4 N>1$ supergravities depends on metric, vectors and scalars. In particular, the action depends on Abelian vectors $\mathcal{A}_{\mu}^{\Lambda}$ via the field strength $F_{\mu \nu}^{\Lambda}=$ $\partial_{\mu} \mathcal{A}_{\nu}^{\Lambda}-\partial_{\nu} \mathcal{A}_{\mu}^{\Lambda}$, on scalars and on the metric ${ }^{1}$

$$
\begin{aligned}
S_{\mathrm{cl}}(F, \phi, g)=\frac{1}{4 \kappa^{2}} \int d^{4} x e( & -\frac{1}{2} R+\operatorname{Im} \mathcal{N}_{\Lambda \Sigma} F_{\mu \nu}^{\Lambda} F^{\mu \nu \Sigma} \\
& \left.+\frac{1}{2 e} \operatorname{Re} \mathcal{N}_{\Lambda \Sigma} \epsilon^{\mu \nu \rho \sigma} F_{\mu \nu}^{\Lambda} F_{\rho \sigma}^{\Sigma}+\frac{1}{2} g_{i j}(\phi) \partial_{\mu} \phi^{i} \partial^{\mu} \phi^{j}\right)
\end{aligned}
$$

Here the kinetic term for vectors $\mathcal{N}_{\Lambda \Sigma}(\phi)$ in general depends on scalars. The matrix $\operatorname{Im} \mathcal{N}_{\Lambda \Sigma}$ is a metric in the vector moduli space. It must be negative definite to provide the positive energy and it must be invertible so that a consistent quantization is possible. These properties of the kinetic matrix $\mathcal{N}_{\Lambda \Sigma}(\phi)$ will be used in the following.

One should keep in mind that the $N=1$ supergravity vector coupling $f_{\alpha \beta}(z)$ corresponds to $-4 i \overline{\mathcal{N}}_{\Lambda \Sigma}$. The manifold of scalars for $3 \leq N \leq 8$ is in each case a unique symmetric coset space $G / H$, where the group $G$ is the Gaillard-Zumino duality symmetry [14], see table 1 . In case $N=2$ it can be a symmetric coset space $G / H$, see table 2 for 7 choices, or it can be also a non-symmetric space described by a Hodge Kähler manifold of $N=2$ special geometry (we will discuss separately the case of $N=2$ hypermultiplets, which are decoupled from the vector multiplets) $[15]-[18-20]$.

The first set of all extended supergravities $d=4 N>1$ based on symmetric coset spaces $G / H$ has a remarkable property that almost all groups $G$ are of type E7 [21-25], see tables 1,2 . This universal property will be used to prove that for all extended supergravities $d=4 N>1$ based on symmetric coset space $G / H$ the vector coupling $\mathcal{N}_{\Lambda \Sigma}(\phi)$ must be scalar dependent, with exception of hyper scalars. In this first set

$$
\frac{\partial}{\partial \phi^{i}} \mathcal{N}_{\Lambda \Sigma}(\phi) \equiv \partial_{i} \mathcal{N}_{\Lambda \Sigma} \neq 0
$$

In the remaining non-symmetric $N=2$ supergravities we will prove that eq. (2.2) is required for a consistency of the special Hodge Kähler manifold. It is quite remarkable

\footnotetext{
${ }^{1}$ In $N=2$ models the kinetic term for (vector multiplet) scalars has a Kähler form: $g_{i \bar{j}} \partial_{\mu} \phi^{i} \partial^{\mu} \bar{\phi}^{\bar{j}}$ with $g_{i \bar{j}}=\partial_{i} \partial_{\bar{j}} K$ and $K$ further restricted in virtue of eq. (2.17), see below.
} 


\begin{tabular}{|c||c|}
\hline$G$ & $\mathbf{R}$ \\
\hline \hline $\mathrm{U}(1, n)$ & $(\mathbf{1}+\mathbf{n})_{\mathbf{c}}$ \\
\hline $\mathrm{SL}(2, \mathbb{R}) \otimes \mathrm{SO}(2, n)$ & $(\mathbf{2}, \mathbf{2}+\mathbf{n})$ \\
\hline $\mathrm{SL}(2, \mathbb{R})$ & $\mathbf{4}$ \\
\hline $\mathrm{Sp}(6, \mathbb{R})$ & $\mathbf{1 4}^{\prime}$ \\
\hline $\mathrm{SU}(3,3)$ & $\mathbf{2 0}$ \\
\hline$S O^{*}(12)$ & $\mathbf{3 2}$ \\
\hline$E_{7(-25)}$ & $\mathbf{5 6}$ \\
\hline
\end{tabular}

Table 2. $N=2$ choices of groups $G$ of the $\frac{G}{H}$ symmetric spaces and their symplectic representations R. The last four lines refer to "magic $N=2$ supergravities".

that when $\partial_{i} \mathcal{N}_{\Lambda \Sigma} \neq 0 N>1$ supergravities require a Pauli coupling of the type (1.4). We will present the details in the $N=2$ case below.

In the cosmological context of creation of matter in the early universe it is important that groups of type E7 do not admit a quadratic bilinear polynomial.

\subsection{Groups of type E7 and compulsory non-minimal vector coupling in $N>1$}

Simply put, groups of type E7 have a symplectic representation $\mathbf{R}$ admitting a symmetric quartic invariant polynomial, but not a quadratic one. We will elaborate further with relevant examples. These were first defined and studied in [21-24]. This was about a decade before $^{2}$ the discovery of supergravity. In fact, surprisingly, all extended supergravities $2 \leq N \leq 8$ described by coset spaces $\frac{G}{H}$ have $G$ of type E7 [25-27], with the exception of $N=2$ group $G=\mathrm{U}(1, n)$ and $N=3$ group $G=\mathrm{U}(3, n)$. These $G=\mathrm{U}(p, n)$ groups are not of type $\mathrm{E} 7$, since they have a primitive quadratic symmetric invariant (in addition to a symplectic bilinear form).

For $N$ between 8 and 3 there are 4 classes of type E7 groups:

$$
E_{7(7)}, \quad S O^{*}(12), \quad \mathrm{SU}(1,5), \quad \mathrm{SL}(2, \mathbb{R}) \otimes \mathrm{SO}(6, n),
$$

and one class $\mathrm{U}(3, n)$ which is not of type E7, see table 1 . In $N=2$ cases of symmetric spaces there are 6 classes of type E7 groups [28], see table 2

$$
E_{7(-25)}, \quad S O^{*}(12), \quad \mathrm{SU}(3,3), \quad \mathrm{Sp}(6, \mathbb{R}), \quad \mathrm{SL}(2, \mathbb{R}), \quad \mathrm{SL}(2, \mathbb{R}) \otimes \mathrm{SO}(2, n),
$$

and one class $\mathrm{U}(1, n)$ which is not of type $\mathrm{E} 7$. Here $n$ is the integer describing the number of matter multiplets for $N=4,3,2$.

Given a symplectic representation $\mathbf{R}$ of dimension $r$

$$
\left(\mathbf{R}_{1}, \mathbf{R}_{2}\right)=-\left(\mathbf{R}_{2}, \mathbf{R}_{1}\right)
$$

one can construct a quartic invariant $q\left(\mathbf{R}_{1}, \mathbf{R}_{2}, \mathbf{R}_{3}, \mathbf{R}_{4}\right)$ which is completely symmetric so that the quartic $G$-invariant polynomial is

$$
\mathcal{I}_{4}(\mathbf{R})=q\left(\mathbf{R}_{1}, \mathbf{R}_{2}, \mathbf{R}_{3}, \mathbf{R}_{4}\right)_{\mathbf{R}_{1}=\mathbf{R}_{2}=\mathbf{R}_{3}=\mathbf{R}_{4}=\mathbf{R}} .
$$

\footnotetext{
${ }^{2}$ The first paper in [21-24] by Brown was submitted in 1967 and published in 1969.
} 
The famous example of such a quartic invariant in $G=E_{7(7)}$ is the Cartan-CremmerJulia invariant $[29,30]$ which defines the area of the $N=8$ black hole horizon [31]. In groups of type $\mathrm{E} 7$ the quadratic symmetric invariant is generically not available, only an antisymmetric one is available, therefore

$$
\left(\mathbf{R}_{1}, \mathbf{R}_{2}\right)_{\mathbf{R}_{1}=\mathbf{R}_{2}}=0
$$

Cases of $N=2$ with $G=\mathrm{U}(1, n)$ and $N=3$ with $G=\mathrm{U}(3, n)$ in fact have a quadratic invariant hermitian form $\left(\mathbf{R}_{1}, \overline{\mathbf{R}}_{2}\right)$ whose imaginary part is the symplectic (antisymmetric) invariant and whose real part is the symmetric quadratic invariant defined as follows (see appendix)

$$
\begin{gathered}
\operatorname{Re}\left(\mathbf{R}_{1}, \overline{\mathbf{R}}_{2}\right)_{\mathbf{R}_{1}=\mathbf{R}_{2}}=\mathcal{I}_{2}(\mathbf{R}) \equiv \sqrt{\left|\mathcal{I}_{4}(\mathbf{R})\right|} \neq 0 \\
\operatorname{Im}\left(\mathbf{R}_{1}, \overline{\mathbf{R}}_{2}\right)_{\mathbf{R}_{1}=\mathbf{R}_{2}}=0
\end{gathered}
$$

Thus, the fundamental representations of pseudounitary groups $U(p, n)$, which have a hermitean quadratic invariant form, do not strictly qualify for groups of type E7. The reason is that they have, in addition to the symplectic invariant, also a symmetric real quadratic invariant instead of a quartic one. This is the reason why from all models of extended supergravities only $G=\mathrm{U}(p, n)$ with $p=1,3$ are not groups of type E7.

Note, however, in all cases with $n \neq 0$ and $N=2,3$ these groups are non-compact and the relevant quadratic invariants can not describe the negative definite vector couplings, as we will see later. In $n=0$ case the groups are compact but there are no scalars. All of this will be important for our consequent analysis. In particular, these $\mathrm{U}(p, n)$ models will form an exceptional case in truncation to $N=1$.

We will now prove here that all vector couplings in extended supergravities with scalars must be non-minimal: they must depend on all scalars with the exception of $N=2$ hypers. For type E7 models this follows from the absence of a symmetric quadratic invariant tensor. For symmetric spaces with $G=\mathrm{U}(p, n)$ it follows from the fact that their symmetric quadratic invariant tensor is not negative definite.

The group $G$ is first embedded into an $\operatorname{Sp}\left(2 n_{v}, \mathbb{R}\right)$ one. The duality symmetry of the theory transforms the symplectic representation as follows

$$
\mathbf{R}^{\prime}=\mathcal{S} \mathbf{R}
$$

where a real symplectic $\operatorname{Sp}\left(2 n_{v}, \mathbb{R}\right)$ matrix is

$$
\mathcal{S}=\left(\begin{array}{ll}
A & B \\
C & D
\end{array}\right), \quad \mathcal{S}^{t} \Omega \mathcal{S}=\Omega, \quad \Omega=\left(\begin{array}{cc}
0 & -\mathbb{I} \\
\mathbb{I} & 0
\end{array}\right),
$$

so that $A^{t} C-C^{t} A=0, B^{t} D-D^{t} B=0, A^{t} D-C^{t} B=1$. The gauge kinetic term $\mathcal{N}$ transforms via fractional transformations

$$
\mathcal{N}^{\prime}=(C+D \mathcal{N})(A+B \mathcal{N})^{-1}
$$


A new symmetric symplectic matrix of dimension $2 n_{v} \times 2 n_{v}$ can be constructed from the $n_{v} \times n_{v}$ matrix $\mathcal{N}$ as follows [32]

$$
\mathcal{M}(\mathcal{N})=\left(\begin{array}{cc}
\operatorname{Im} \mathcal{N}+\operatorname{Re} \mathcal{N}(\operatorname{Im} \mathcal{N})^{-1} \operatorname{Re} \mathcal{N} & -\operatorname{Re} \mathcal{N}(\operatorname{Im} \mathcal{N})^{-1} \\
-(\operatorname{Im} \mathcal{N})^{-1} \operatorname{Re} \mathcal{N} & (\operatorname{Im} \mathcal{N})^{-1}
\end{array}\right)
$$

such that

$$
\mathcal{M}^{T} \Omega \mathcal{M}=\Omega, \quad \mathcal{M}^{T}=\mathcal{M} .
$$

This matrix transforms as a tensor under duality transformations. If $\mathcal{N}$ would be constant, $\mathcal{M}$ would also be constant. This would imply that there is a symmetric invariant tensor of the group $G$. However, none of these theories with E7 type $G$ has an invariant symmetric quadratic form for the symplectic representation $\mathbf{R}$ of $G$, by definition. The only extended supergravities, which have a symmetric quadratic invariant are the $N=3$ and $N=2$ theories with $\mathrm{U}(p, n)$ groups. However, their quadratic real form does not have all negative eigenvalues but has a Lorentzian structure, having the signature $(p, n)$, due to the noncompactness of $G$ for $n \geq 1$ : $\mathrm{U}(3, n)$ for $N=3$ and $\mathrm{U}(1, n)$ for $N=2$, see the first row in table 1 and table 2, respectively. Note that $\operatorname{Im} \mathcal{N}$ and $\mathcal{M}$ must be negative definite: for $\operatorname{Im} \mathcal{N}$ this is a condition for positive energy, for $\mathcal{M}$ the proof can be found in [18-20]. Thus, only in absence of scalars when $n=0$ in $\mathrm{U}(3, n)$ and $\mathrm{U}(1, n)$ models the constant matrix $\mathcal{M}$ exist, but they are irrelevant since there are no scalars and the Gaillard-Zumino duality group [14] becomes a compact group $\mathrm{U}(p)$. This proves eq. (2.2) for all scalar-dependent $N>2$ supergravities based on coset spaces.

\section{$2.2 \quad N=2$}

The case of $N=2$ requires a separate study since scalars may belong to either vector multiplets or hypermultiplets [15, 18-20]. Hypermultiplets and vector multiplets are decoupled: the interactions of hyper multiplets are described by a quaternionic geometry whereas the interactions of the vector multiplets are described by the special geometry. Therefore in $N>2$

$$
\frac{\partial}{\partial \phi^{i}} \mathcal{N}_{\Lambda \Sigma}(\phi) \neq 0 \quad \text { for all scalars }
$$

whereas in $N=2$ the kinetic term of vectors depends on all scalars from the vector multiplets $\phi_{v}^{i}$ and does not depend on any scalars from the hypermultiplets $q^{u}$

$$
\frac{\partial}{\partial \phi_{v}^{i}} \mathcal{N}_{\Lambda \Sigma}(\phi) \neq 0, \quad \frac{\partial}{\partial q^{u}} \mathcal{N}_{\Lambda \Sigma}(\phi)=0
$$

\subsection{1 $N=2$ special geometry and compulsory non-minimal vector coupling}

In $N=2$ special geometry [15]-[18-20] the Riemann tensor of the Kähler manifold is

$$
R_{i \bar{j} k \bar{l}}=-\left(g_{i \bar{j}} g_{k \bar{l}}+g_{i \bar{l}} g_{k \bar{j}}\right)+C_{i k p} C_{\bar{j} \bar{l} \bar{p}} g^{p \bar{p}}
$$


where $C_{i j k}$ is a covariantly holomorphic 3 -form defined on a Hodge Kähler manifold. The theory is based on covariantly holomorphic symplectic section $\left(L^{\Lambda}, M_{\Lambda}\right)$. We also define $f_{i}^{\Lambda}=D_{i} L^{\Lambda}, h_{i \Lambda}=D_{i} M_{\Lambda}$. The Kähler metric in these notation is

$$
g_{i \bar{j}}=-2 \operatorname{Im} \mathcal{N}_{\Lambda \Sigma} f_{i}^{\Lambda} \bar{f}_{\bar{j}}^{\Sigma}
$$

and the $C_{i k j}$ tensor is

$$
C_{i j k}=f_{i}^{\Lambda} \partial_{j} \overline{\mathcal{N}}_{\Lambda \Sigma} f_{k}^{\Sigma}
$$

Here $\mathcal{N}_{\Lambda \Sigma}$ is the vector kinetic matrix, providing the following relations:

$$
M_{\Lambda}=\mathcal{N}_{\Lambda \Sigma} L^{\Sigma}, \quad \mathcal{D}_{i} M_{\Lambda}=\overline{\mathcal{N}}_{\Lambda \Sigma} \mathcal{D}_{i} L^{\Sigma} .
$$

It follows that

$$
\partial_{i} \mathcal{N}_{\Lambda \Sigma} L^{\Sigma}=-(\mathcal{N}-\overline{\mathcal{N}})_{\Lambda \Sigma} f_{i}^{\Sigma}
$$

The Pauli terms with non-minimal vector couplings in $N=2$ supergravity are

$$
C_{i j k} \bar{\lambda}^{i A} \sigma_{\mu \nu} \lambda^{j B} F^{k \mu \nu} \epsilon_{A B}=\partial_{j} \overline{\mathcal{N}}_{\Lambda \Sigma} \bar{\lambda}^{\Lambda A} \sigma_{\mu \nu} \lambda^{i B} F^{\Sigma \mu \nu} \epsilon_{A B}
$$

and for non-vanishing $\partial_{i} \overline{\mathcal{N}}_{\Lambda \Sigma}$ they remain upon a consistent truncation in the corresponding version of $N=1$ supergravity in agreement with eq. (1.4).

For constant $\mathcal{N}_{\Lambda \Sigma}$ eq. (2.21) implies, since $\operatorname{Im} \mathcal{N}_{\Lambda \Sigma}$ must be invertible for a consistent quantization of supergravity, that

$$
f_{i}^{\Lambda}=0 .
$$

In such case it follows from eq. (2.18) that

$$
g_{i \bar{j}}=0 .
$$

Also for constant $\mathcal{N}_{\Lambda \Sigma}$ eq. (2.19) implies that $C_{i j k}=0$. The vanishing metric of the Kähler geometry $g_{i \bar{j}}=0$ means that there are no vector multiplet scalars. In fact, $\partial_{i} \mathcal{N}_{\Lambda \Sigma}=0$ in $N>1$ is only possible if there are no scalars, except for hypers in $N=2$ case. For example, $N=3$ models of the type defined in [33-35] can be reduced to $N=2$ models with vector multiplets with $C_{i j k}=0$ and hypermultiplets. When further reduced to $N=1$ these models have only minimal kinetic terms [36].

We conclude therefore that eq. (2.2) is compulsory for a consistent $N=2$ special geometry. We remind that in this case we only used properties of special geometry which encompass both symmetric and non-symmetric special Kähler manifolds.

$N=2$ non-symmetric space models of special geometry also universally require a nonminimal vector coupling. These couplings remain non-minimal and scalar dependent, when truncated to $N=1$, provided that $C_{i j k} \neq 0$. So, special geometry with a non-vanishing $C_{i j k}$ tensor can be regarded as a generalization of groups of type E7 for non-symmetric special manifolds, see the appendix for the details.

It is particularly interesting that the simplest cases of non-symmetric spaces with a non-vanishing $C_{i j k}$ correspond to the Calabi-Yau special geometries, as they come in type II string theory compactifications [37]. The related c-map hypergeometry is also nonsymmetric (although it is an Einstein space). It may also be useful to remind here that the examples of $10^{500}$ string landscape vacua [38] are based on such Calabi-Yau threefold with the non-vanishing triple intersection numbers $C_{i j k}$. 


\section{$2.3 \quad$ All kinetic terms in $N>1$}

We have focused our attention to the fact that the vector kinetic terms are non-minimal since we are interested in cosmological applications of the vertices which allow the decay of the inflaton and unwanted relics into particles of the standard model. However, we may add here that all kinetic terms in $N>1$ supergravities are "non-minimal" which means that they depend on scalars. For supergravity manifolds of $N>2$ this is obvious because they are Einstein spaces with (negative) constant curvature.

For $N=2$ theories vector multiplets are coupled to special geometry space which is never flat, while hypermultiplets are described by quaternionic geometries which are generically not symmetric and belong to Einstein spaces with constant negative curvature [39]. This means that the kinetic terms for scalars in all cases of $N=2$ are non-minimal, however, the vector kinetic matrix depends only on scalars from the vector multiplets and does not depend on hypers.

\section{Consistent truncations of $N>1$ to $N=1$}

A detailed study of the consistent truncation of $N>1$ supergravity to an $N=1$ was performed in [40-42]. Here we would like to ask whether a theory with $N>1$ can be consistently truncated to an $N=1$ supergravity with minimally coupled vectors. This is a very strong constraint since it would require not only the matrix $\mathcal{N}$ to become holomorphic, but in fact constant. For symmetric spaces this is only possible in one particular case if we consider the $C P^{n}$ models with $C_{i j k}=0$, keep $n_{c}$ chiral multiplets and $n_{v}=n-n_{c}$ vector multiplets. These models may also include some hypermultiplets decoupled from vector multiplets. Then the reduced theory will have a constant $\mathcal{N}$ matrix.

For $N \geq 4$ consistent truncations to $N=2$ exist which include, beyond vector multiplets, also hypermultiplets (see f.e. table 1 in [43]). Then further reduced to $N=1$ hyperscalars will be minimally coupled to vectors. However, the scalar sector coming from vectors will not be minimally coupled, since in general $C_{i j k} \neq 0$. There is only a universal truncation to $N=1$ which is minimally coupled which is the axion-dilaton $N=2$ multiplet further truncated to a $N=1$ vector multiplet. We observe that this is always possible because this corresponds to the $N=1$ truncation of a decoupled space for a $\frac{\mathrm{SL}(2, \mathbb{R})}{\mathrm{U}(1)} \times \frac{\mathrm{SO}(2, n)}{\mathrm{SO}(2) \times \mathrm{SO}(n)}$ manifold when we set $n=0$.

We then conclude that minimally coupled scalars in $N=1$ truncation from higher $N$ can only be obtained if, at the level of $N=2$ the scalars come from hypermultiplets or from $C P^{n}$ submanifolds of the $N=2$ manifolds (the axion-dilaton $\mathrm{N}=2$ multiplet corresponds to the $C P^{1}$ case in which case no scalar is left from this sector).

Following [40-42] the $\mathcal{N}_{\Lambda \Sigma}$ matrix in $N=2$ is

$$
\mathcal{N}_{\Lambda \Sigma}=\bar{F}_{\Lambda \Sigma}-2 i \bar{T}_{\Lambda} \bar{T}_{\Sigma}\left(L^{Z} \operatorname{Im} F_{Z W} L^{W}\right),
$$

where $F_{Z W}$ is the holomorphic second derivative of the prepotential, $L^{Z}$ is the relevant part of the covariantly holomorphic section and $T_{\Lambda}$ are the projectors on the graviphoton. 
We now redefine the indices so that the original $\Lambda$ taking values $0,1, \ldots, n$ is split into an index $\alpha$ taking values $1, \ldots, n_{v}$ for $N=1$ vector multiplets and an index $X$ taking values in $0,1, \ldots, n_{c}$. Here $n=n_{c}+n_{v}$. Note that the $N=1$ vector index $\alpha$ does not include 0 .

A consistent truncation to $N=1$ from $N=2$ models with $C_{i j k}=0$ means that one starts with the quadratic holomorphic prepotential $F(X)$. In such case one finds from (3.1) that

$$
\mathcal{N}_{\alpha \beta}=\bar{F}_{\alpha \beta}=-i \delta_{\alpha \beta}
$$

and $\operatorname{Im} \mathcal{N}<0$ as it should be. Here the relation between the $N=2$ and $N=1$ kinetic matrix is $\bar{F}_{\alpha \beta}=\mathcal{N}_{\alpha \beta}=-\frac{i}{4} \bar{f}_{\alpha \beta}(\bar{z})$ which agrees with [3].

Generic models derived from higher dimensions ${ }^{3}$ have $C_{i j k} \neq 0$. Therefore the nonminimal vector coupling $\mathcal{N}_{\Lambda \Sigma}$ reduced to $N=1$ will not be constant, in general. As an example one can consider the effective action of type $N=1$ Calabi-Yau orientifolds derived from the compactification of string theory in [44-46]. This is a generic case of superstring inspired $N=1$ supergravities which arise from compactified Calabi-Yau orientifolds. They produce the cubic coupling associated with the holomorphic vector coupling

$$
\overline{\mathcal{N}}_{\alpha \beta}(z)=d_{\alpha \beta p} z^{p} .
$$

Here as above $\alpha, \beta=1, \ldots, n_{v}$ and $p=1, \ldots, n_{c}$ and $d_{\alpha \beta p}$ codifies the Calabi-Yau threefolds intersection numbers. The kinetic holomorphic vector coupling matrix is linear in the scalars of a chiral multiplets $z^{p}=a^{p}+i \varphi^{p}$ and is proportional to

$$
d_{\alpha \beta p} \varphi^{p} F_{\mu \nu}^{\alpha} F^{\beta \mu \nu}+d_{\alpha \beta p} a^{p} F_{\mu \nu}^{\alpha} \tilde{F}^{\beta \mu \nu} .
$$

Thus, if one of the scalars in the chiral multiplet $z^{p}$ or their combination is an inflaton field or any other heavy scalar we would like to get rid of, it has a cubic vertex and a route of decay into two vectors. In the same models there is a Pauli interaction of the following form, see eq. (1.4)

$$
d_{\alpha \beta p} \bar{\chi}^{p} \sigma_{\mu \nu} \lambda^{\alpha} F^{\beta \mu \nu} .
$$

If the fermion partner of the inflaton or any other heavy fermion was created at the nonperturbative stage of preheating, it will also decay into a gaugino and a vector field at a later stage via the compulsory Pauli interaction.

\section{Discussion}

The minimal $N=1$ supergravity seems to be the simplest one and described in [4] with the minimal vector coupling (1.2). It may be viewed, however, as an incomplete theory, when the creation of matter in the Universe after inflation is studied. In this paper we have argued that any version of $N=1$ supergravity inspired by either supergravities with more supersymmetries (extended $N>1$ supergravities), or originating from higher dimensions and compactifications of superstring theory, must have a non-minimal scalar dependent

\footnotetext{
${ }^{3}$ With exception of the string theory derivation of $N=3$ supergravity model with $\mathrm{U}(3, n)$ duality group based on a $\frac{T^{6}}{Z^{2}}$ orientifold [35].
} 
vector couplings, as well as Pauli couplings. In non-supersymmetric theories the coupling $a F \tilde{F}$ of vectors with axion field $a(x)$ was studied intensely in QFT and in applications to cosmology, see for example [47] and references therein. The couplings of vectors to moduli fields $e^{\phi} F F$ were also studied in various situations in QFT and cosmology.

In this paper we proved that in $N=1$ supergravity obtained via truncation from extended $N>1$ supergravities, or originating from higher dimensions and compactifications of superstring theory, the interactions between vectors and scalars and the corresponding Pauli interactions are compulsory (with exception of $\mathrm{U}(p, n)$ models and hyperscalars), namely $N=1$ action includes the following interactions:

$$
\operatorname{Im} \mathcal{N}_{\Lambda \Sigma}(\phi) F_{\mu \nu}^{\Lambda} F^{\mu \nu \Sigma}+\operatorname{Re} \mathcal{N}_{\Lambda \Sigma}(\phi) F_{\mu \nu}^{\Lambda} \tilde{F}^{\mu \nu \Sigma}+\frac{\partial \mathcal{N}_{\Lambda \Sigma}}{\partial \phi^{p}} \bar{\chi}^{p} \sigma_{\mu \nu} \lambda^{\Lambda} F^{\Sigma \mu \nu}+\ldots
$$

When the kinetic function $\mathcal{N}_{\Lambda \Sigma}(\phi)$ is linear in scalars

$$
\overline{\mathcal{N}}_{\Lambda \Sigma}(\phi)=d_{\Lambda \Sigma p} z^{p}
$$

and $d_{\Lambda \Sigma p}$ are some constants (which have a simple interpretation in Calabi-Yau compactifications or special geometry in $N=2$ ), the theory has a cubic coupling between scalars and vectors. If the scalar is an inflaton or some heavy scalar it has a simple route of decay via creation of vector particles, which in turn couple to other standard model particles and create the whole matter in the universe. Some unwanted fermions, partners of inflaton or of the heavy scalars, will also decay via the cubic Pauli coupling.

A remarkable feature of $N>1$ supergravities is the fact that the non-minimal vector couplings and Pauli couplings are compulsory and universal (with exception of models in $N=2$ which have only hyper scalars). One quick look at tables 1 and 2, which give the list of duality symmetries $G$ of all symmetric space $G / H$ supergravities, shows how different they are and how distinct are their duality groups $G$. The non-symmetric spaces of $N=2$ special geometry have enormous amount of choices associated with the non-vanishing 3 -forms $C_{i j k}$. The proof of non-minimal vector couplings for all $N>1$ symmetric as well as non-symmetric supergravities is based on the universal feature of all of these models: they are based on duality groups of type E7. The proof follows from the definition of these groups.

Exceptional cases of $\mathrm{U}(p, n), p=3,1$ groups, which are not of type E7, also require a non-minimal vector coupling in $N>1$ models, the proof being based on the non-compactness of these groups for $n \neq 0$ and negative definiteness of the kinetic term for vectors.

The vector couplings remain non-minimal, scalar dependent, when truncated to $N=$ 1 for all models of type E7, which form a majority of extended supergravity models. These couplings become minimal, scalar independent, in the exceptional case $\mathrm{U}(p, n)$, $p=3,1$, with vanishing 3 -forms $C_{i j k}=0$ in $N=2$ case, or when all scalars originate from the hypers.

This universality of a scalar dependent vector coupling may have a cosmological significance: it suggests that creation of matter in the universe via non-minimal vector and Pauli couplings may be a dominant factor in theories inspired by superstring theory and extended supergravity. 


\section{Acknowledgments}

It is a pleasure to thank R. Bond, J. Broedel, J.J. Carrasco, A. Linde, L. McAllister, K. Olive and E. Silverstein for stimulating conversations. The work of S.F. has been supported by the ERC Advanced Grant no. 226455, "Supersymmetry, Quantum Gravity and Gauge Fields" (SUPERFIELDS), and in part by DOE Grant DE-FG03-91ER40662. The work of R.K. was supported by the Stanford Institute of Theoretical Physics and NSF grant 0756174 .

\section{A Special geometry and groups of type E7}

The contact of special geometry with groups of type E7 can be made by introducing a quartic functional of central charge $Z$ and matter charges $Z_{i}=D_{i} Z$, as shown in [48]

$$
\mathcal{I}(\phi, Q)=\left(Z \bar{Z}-Z_{i} \bar{Z}^{i}\right)^{2}+\frac{2}{3} i\left(Z N_{3}(\bar{Z})-\bar{Z} \bar{N}_{3}(Z)\right)-g^{i \bar{i}} C_{i j k} C_{\bar{i} \bar{j} \bar{k}} \bar{Z}^{j} \bar{Z}^{k} Z^{\bar{j}} Z^{\bar{k}} .
$$

The quartic invariant $\mathcal{I}(\phi, Q)$ is actually the coordinate covariant expression of the " $\mathrm{h}$ " function introduced in eq. (2.31) of [49] if one replaces (half of) the quaternionic coordinates with the dyonic charge vector $Q$, in the c-map construction of [37].

This quartic invariant has the property that at the attractor points

$$
2 \bar{Z} Z_{i}+i C_{i j k} \bar{Z}^{j} \bar{Z}^{k}=0
$$

it takes the following value

$$
\mathcal{I}\left(\phi_{H}, Q\right)=V_{H}^{2}-\frac{32}{3}|Z|^{2}\left(Z_{i} \bar{Z}^{i}\right)_{H}
$$

where $V_{H}$ is the black hole potential (at the attractor point) at the black hole horizon [26, 27]. As a result, either

$$
\mathcal{I}\left(\phi_{H}, Q\right)=\mathcal{I}_{4}(Q)
$$

for symmetric special geometries of E7 type, or

$$
\mathcal{I}\left(\phi_{H}, Q\right)=\left(\mathcal{I}_{2}(Q)\right)^{2}
$$

for symmetric geometries with $C_{i j k}=0$, i.e. $\frac{\mathrm{U}(1, n)}{\mathrm{U}(1) \otimes \mathrm{U}(n)} C P^{n}$ models. Therefore, as stated in the text, in $N=2$ groups of type E7 require $C_{i j k} \neq 0$, which is the condition for the existence of the "primitive quartic invariant".

For non-symmetric spaces the quartic functional exists (as shown in eq. $(4.2)$ of $[26,27]$ ) however, it is not invariant as it is scalar dependent. Note that the case $C_{i j k}=0$ is the only case which can give a consistent truncation to the minimally coupled $N=1$ theories.

Open Access. This article is distributed under the terms of the Creative Commons Attribution Noncommercial License which permits any noncommercial use, distribution, and reproduction in any medium, provided the original author(s) and source are credited. 


\section{References}

[1] L. Kofman, A.D. Linde and A.A. Starobinsky, Reheating after inflation, Phys. Rev. Lett. 73 (1994) 3195 [hep-th/9405187].

[2] L. Kofman, A.D. Linde and A.A. Starobinsky, Towards the theory of reheating after inflation, Phys. Rev. D 56 (1997) 3258 [hep-ph/9704452].

[3] E. Cremmer, S. Ferrara, L. Girardello and A. Van Proeyen, Yang-Mills theories with local supersymmetry: Lagrangian, transformation laws and superHiggs effect, Nucl. Phys. B 212 (1983) 413 [inSPIRE].

[4] J. Wess, J. Bagger, Supersymmetry and supergravity, Princeton University Press, Princeton U.S.A. (1992).

[5] R. Kallosh, A. Linde, K.A. Olive and T. Rube, Chaotic inflation and supersymmetry breaking, Phys. Rev. D 84 (2011) 083519 [arXiv:1106.6025] [INSPIRE].

[6] R. Kallosh and A. Linde, New models of chaotic inflation in supergravity, JCAP 11 (2010) 011 [arXiv: 1008.3375] [INSPIRE].

[7] R. Kallosh, A. Linde and T. Rube, General inflaton potentials in supergravity, Phys. Rev. D 83 (2011) 043507 [arXiv: 1011.5945] [InSPIRE].

[8] J. Blanco-Pillado, C. Burgess, J.M. Cline, C. Escoda, M. Gomez-Reino, et al., Racetrack inflation, JHEP 11 (2004) 063 [hep-th/0406230] [INSPIRE].

[9] J.P. Conlon and F. Quevedo, Astrophysical and cosmological implications of large volume string compactifications, JCAP 08 (2007) 019 [arXiv:0705.3460] [INSPIRE].

[10] N. Barnaby, J. Bond, Z. Huang and L. Kofman, Preheating after modular inflation, JCAP 12 (2009) 021 [arXiv: 0909.0503] [INSPIRE].

[11] J. Braden, L. Kofman and N. Barnaby, Reheating the universe after multi-field inflation, JCAP 07 (2010) 016 [arXiv: 1005.2196] [INSPIRE].

[12] M. Endo, M. Kawasaki, F. Takahashi and T. Yanagida, Inflaton decay through supergravity effects, Phys. Lett. B 642 (2006) 518 [hep-ph/0607170] [INSPIRE].

[13] M. Endo, K. Kadota, K.A. Olive, F. Takahashi and T. Yanagida, The decay of the inflaton in no-scale supergravity, JCAP 02 (2007) 018 [hep-ph/0612263] [INSPIRE].

[14] M.K. Gaillard and B. Zumino, Duality rotations for interacting fields, Nucl. Phys. B 193 (1981) 221 [inSPIRE].

[15] B. de Wit and A. Van Proeyen, Potentials and symmetries of general gauged $N=2$ supergravity: Yang-Mills models, Nucl. Phys. B 245 (1984) 89 [INSPIRE].

[16] E. Cremmer and A. Van Proeyen, Classification of Kähler manifolds in $N=2$ vector multiplet supergravity couplings, Class. Quant. Grav. 2 (1985) 445 [INSPIRE].

[17] A. Strominger, Special geometry, Commun. Math. Phys. 133 (1990) 163 [INSPIRE].

[18] A. Ceresole, R. D'Auria and S. Ferrara, The symplectic structure of $N=2$ supergravity and its central extension, Nucl. Phys. Proc. Suppl. 46 (1996) 67 [hep-th/9509160] [INSPIRE].

[19] L. Andrianopoli, M. Bertolini, A. Ceresole, R. D'Auria, S. Ferrara, et al., $N=2$ supergravity and $N=2$ super Yang-Mills theory on general scalar manifolds: symplectic covariance, gaugings and the momentum map, J. Geom. Phys. 23 (1997) 111 [hep-th/9605032] [INSPIRE]. 
[20] L. Andrianopoli, R. D'Auria and S. Ferrara, U duality and central charges in various dimensions revisited, Int. J. Mod. Phys. A 13 (1998) 431 [hep-th/9612105] [InSPIRE].

[21] R.B. Brown, Groups of type E7, J. fur die reine und angewandte Mathematik 236 (1969) 79.

[22] V.G. Kac Some remarks on nilpotent orbits, J. Alg. 64 (1980) 190.

[23] J.F. Adams Lectures on exceptional Lie algebras, Chicago Lectures in Mathematical Series, J.P. May et al. eds. (1984).

[24] S. Krutelevich, Jordan algebras, exceptional groups and higher composition laws, arXiv:math/0411104.

[25] L. Borsten, D. Dahanayake, M. Duff and W. Rubens, Black holes admitting a Freudenthal dual, Phys. Rev. D 80 (2009) 026003 [arXiv:0903.5517] [INSPIRE].

[26] S. Ferrara, A. Marrani and A. Yeranyan, Freudenthal duality and generalized special geometry, Phys. Lett. B 701 (2011) 640 [arXiv:1102.4857] [INSPIRE].

[27] L. Borsten, M. Duff, S. Ferrara, A. Marrani and W. Rubens, Small orbits, arXiv:1108.0424 [INSPIRE].

[28] M. Günaydin, G. Sierra and P. Townsend, Exceptional supergravity theories and the magic square, Phys. Lett. B 133 (1983) 72 [inSPIRE].

[29] E. Cartan, Euvres complètes, Editions du Centre National de la Recherche Scientifique, Paris France (1984).

[30] E. Cremmer and B. Julia, The SO(8) supergravity, Nucl. Phys. B 159 (1979) 141 [InSPIRE].

[31] R. Kallosh and B. Kol, E $E_{7}$ symmetric area of the black hole horizon, Phys. Rev. D 53 (1996) 5344 [hep-th/9602014] [INSPIRE].

[32] P. Breitenlohner, D. Maison and G.W. Gibbons, Four-Dimensional black holes from Kaluza-Klein theories, Commun. Math. Phys. 120 (1988) 295 [INSPIRE].

[33] S. Ferrara and C. Kounnas, Extended supersymmetry in four-dimensional type II strings, Nucl. Phys. B 328 (1989) 406 [INSPIRE].

[34] C. Kounnas and A. Kumar, BPS states in $N=3$ superstrings, Nucl. Phys. B 511 (1998) 216 [hep-th/9709061] [INSPIRE].

[35] A.R. Frey and J. Polchinski, N=3 warped compactifications, Phys. Rev. D 65 (2002) 126009 [hep-th/0201029] [INSPIRE].

[36] L. Andrianopoli, R. D'Auria, S. Ferrara and M. Lledó, $N=2$ superHiggs, $N=1$ Poincaré vacua and quaternionic geometry, JHEP 01 (2003) 045 [hep-th/0212236] [INSPIRE].

[37] S. Cecotti, S. Ferrara and L. Girardello, Geometry of type II superstrings and the moduli of superconformal field theories, Int. J. Mod. Phys. A 4 (1989) 2475 [inSPIRE].

[38] F. Denef, M.R. Douglas and B. Florea, Building a better racetrack, JHEP 06 (2004) 034 [hep-th/0404257] [INSPIRE].

[39] J. Bagger and E. Witten, Matter couplings in $N=2$ supergravity, Nucl. Phys. B 222 (1983) 1 [inSPIRE].

[40] L. Andrianopoli, R. D'Auria and S. Ferrara, Supersymmetry reduction of $N$ extended supergravities in four-dimensions, JHEP 03 (2002) 025 [hep-th/0110277] [INSPIRE]. 
[41] L. Andrianopoli, R. D'Auria and S. Ferrara, Consistent reduction of $N=2 \rightarrow N=1$ four-dimensional supergravity coupled to matter, Nucl. Phys. B 628 (2002) 387 [hep-th/0112192] [INSPIRE].

[42] L. Andrianopoli, R. D'Auria, S. Ferrara and M. Trigiante, Black-hole attractors in $N=1$ supergravity, JHEP 07 (2007) 019 [hep-th/0703178] [INSPIRE].

[43] S. Ferrara and A. Marrani, $N=8$ non-BPS attractors, fixed scalars and magic supergravities, Nucl. Phys. B 788 (2008) 63 [arXiv:0705.3866] [InSPIRE].

[44] T.W. Grimm and J. Louis, The effective action of $N=1$ Calabi-Yau orientifolds, Nucl. Phys. B 699 (2004) 387 [hep-th/0403067] [INSPIRE].

[45] T.W. Grimm and J. Louis, The effective action of type IIA Calabi-Yau orientifolds, Nucl. Phys. B 718 (2005) 153 [hep-th/0412277] [INSPIRE].

[46] R. Blumenhagen, B. Körs, D. Lüst and S. Stieberger, Four-dimensional string compactifications with D-branes, orientifolds and fluxes, Phys. Rept. 445 (2007) 1 [hep-th/0610327] [INSPIRE].

[47] N. Barnaby, E. Pajer and M. Peloso, Gauge field production in axion inflation: consequences for monodromy, non-Gaussianity in the CMB and gravitational waves at interferometers, arXiv:1110.3327 [INSPIRE].

[48] B.L. Cerchiai, S. Ferrara, A. Marrani and B. Zumino, Duality, entropy and ADM mass in supergravity, Phys. Rev. D 79 (2009) 125010 [arXiv:0902.3973] [INSPIRE].

[49] B. de Wit, F. Vanderseypen and A. Van Proeyen, Symmetry structure of special geometries, Nucl. Phys. B 400 (1993) 463 [hep-th/9210068] [INSPIRE]. 